\begin{tabular}{|c|c|}
\hline Title & Layer-selective spin amplification in size modul ated quantum nanocolumn \\
\hline Author(s) & Hiura, Satoshi; Saito, Shotaro; Takay ama, Junichi; Kiba, Takay uki; Murayama, A kihiro \\
\hline Citation & $\begin{array}{l}\text { A pplied physics letters, 115(1), } 013102 \\
\text { https://doi.org/10.1063/1.5098110 }\end{array}$ \\
\hline Issue Date & 2019-07-01 \\
\hline DOC URL & http:/hdl .handle.net/2115/78802 \\
\hline Rights & $\begin{array}{l}\text { This article may be downloaded for personal use only. A ny other use requires prior permission of the author and AIP } \\
\text { Publishing. This article appeared in A ppl. Phys. Lett. 115(1), } 013102 \text { (2019) and may be found at } \\
\text { http:/doi.org/10.1063/1.5098110. }\end{array}$ \\
\hline Type & article \\
\hline File Information & 1.5098110.pdf \\
\hline
\end{tabular}

Instructions for use 


\section{Layer-selective spin amplification in size- modulated quantum nanocolumn}

Cite as: Appl. Phys. Lett. 115, 013102 (2019); https://doi.org/10.1063/1.5098110

Submitted: 01 April 2019. Accepted: 19 June 2019. Published Online: 02 July 2019

Satoshi Hiura (D), Shotaro Saito, Junichi Takayama (D), Takayuki Kiba (D), and Akihiro Murayama


Export Citation

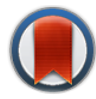

View Online

CrossMark

\section{ARTICLES YOU MAY BE INTERESTED IN}

Accurate measurement of nanomechanical motion in a fiber-taper nano-optomechanical system

Applied Physics Letters 115, 013104 (2019); https://doi.org/10.1063/1.5110272

High-performance monolayer $\mathrm{MoS}_{2}$ field-effect transistor with large-scale nitrogen-doped graphene electrodes for Ohmic contact

Applied Physics Letters 115, 012104 (2019); https://doi.org/10.1063/1.5094682

Bright electroluminescence in ambient conditions from WSe $2 \mathrm{p}-\mathrm{n}$ diodes using pulsed injection

Applied Physics Letters 115, 011103 (2019); https://doi.org/10.1063/1.5100306

\section{Applied Physics Letters}

Mid-IR and THz frequency combs special collection

Read Now! 


\title{
Layer-selective spin amplification in size-modulated quantum nanocolumn
}

\author{
Cite as: Appl. Phys. Lett. 115, 013102 (2019); doi: 10.1063/1.5098110 \\ Submitted: 1 April 2019 - Accepted: 19 June 2019 . \\ Published Online: 2 July 2019
}



\author{
Satoshi Hiura, ${ }^{1, a)}$ (D) Shotaro Saito, Junichi Takayama,' (D) Takayuki Kiba, ${ }^{2}$ (D) and Akihiro Murayama' \\ AFFILIATIONS \\ ${ }^{7}$ Faculty of Information Science and Technology, Hokkaido University, Kita 14, Nishi 9, Kita-ku, Sapporo 060-0814, Japan \\ ${ }^{2}$ Kitami Institute of Technology, 165 Koen-cho, Kitami 090-8507, Japan

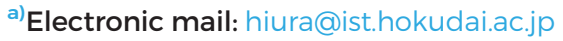

\begin{abstract}
The optical spin properties of size-modulated quantum nanocolumns (QNCs), which are composed of 9 layers of vertically coupled InGaAs quantum dots (QDs), have been studied by circularly polarized time-resolved photoluminescence spectroscopy of QD excited states with barrier excitation. High spin polarization at the emissive state is one of the essential elements in the development of spin-functional optical devices. Coupling of QD excited states can enhance the spin polarization if only minority spins are effectively removed from the emissive excited states. In this study, size-modulated QNCs with the increasing size toward the upper layer were grown, and we revealed that the combination of QD size modulation and electron wavefunction coupling in the stacking direction can greatly enhance spin polarization during light emission from the smaller-sized QD layers. We observed a temporal spin amplification of more than $80 \%$ at coupled excited states. This enhancement is derived from the size-modulation-induced selective transfer of minority spins to the larger-sized QD layers, which have abundant excited states where electron spins are transferred. In addition, we found that QNCs can retain high spin polarization even at high excitation spin density. Our findings of spin amplification during light emission will provide QNC systems suitable for spin-functional optical devices.
\end{abstract}

Published under license by AIP Publishing. https://doi.org/10.1063/1.5098110

\begin{abstract}
Optospintronics is a fascinating research field with high potential for the development of optical spintronic devices. Many works on spin-polarized light emitting diodes ${ }^{1}$ and spin photodiodes ${ }^{2,3}$ have been conducted. In optospintronics, retention of spin information is a key technology. III-V compound semiconductor quantum dots (QDs) can retain the spin state for longer than a nanosecond. ${ }^{4,5}$ Therefore, spin-functional optical devices using QDs as active layers have attracted much attention. High-density QDs are important for the increase in emission intensity and optical gain. ${ }^{6}$ For spin-dependent properties, high-density QDs can suppress the spin-state filling effect, which degenerates the spin polarization at emissive states. ${ }^{7,8}$ They also cause an electronic coupling among QDs. Spin relaxation can be temporally suppressed at coupled excited states (ESs) owing to the selective transfer of minority spins among laterally aligned QDs. ${ }^{9,10}$ Multilayer QDs are also effective for increasing QD density. By decreasing the spacer thickness, electron wavefunctions are coupled among vertically aligned QDs. ${ }^{11}$ When the spacer layer becomes thinner, the strain field induced by the buried QDs causes earlier formation of the second layer of QDs. ${ }^{12,13}$ This reduces the actual spacer thickness $^{14,15}$ and increases the QD size ${ }^{13,14}$ along the stacking direction. As a result, vertically aligned QDs are formed with the increasing size toward the upper layer. This structure can be referred to as a size-
\end{abstract}

modulated quantum nanocolumn (QNC). This nanosystem can provide significant insights into spin amplification induced by spin transfer within QNCs.

In this study, we grew size-modulated QNCs consisting of 9 layers of QDs. The QNC shows a temporal spin amplification of more than $80 \%$ at coupled excited states. This high spin polarization is attributed to the selective transfer of minority spins from the lowerlying emissive layers to the upper-lying nonemissive layers. In addition, we found that the QNC can retain high spin polarization even at high excitation spin density.

1,3 , and 9 layers of $\operatorname{In}_{0.5} \mathrm{Ga}_{0.5}$ As QDs with a nominal thickness of $2 \mathrm{~nm}$ were grown on $\mathrm{GaAs}(100)$ substrates under growth conditions similar to those used previously. ${ }^{7-9}$ For the multilayer QDs, the spacer thickness was set to $10 \mathrm{~nm}$ and the InGaAs supply amount was the same for the growth of all QD layers. The additional QD layer was grown on the $40 \mathrm{~nm}$-thick capping layer to observe the QD structure by atomic force microscopy (AFM). The obtained structure corresponds to the first layer. Transmission electron microscopy (TEM) measurements were also performed to investigate the QD structure in the stacking direction. Circularly polarized photoluminescence (PL) and its time-resolved measurements were performed at $6 \mathrm{~K}$ using a method described previously. ${ }^{7-9}$ In this PL measurement, the 
excitation light was incident on the sample surface and the PL emission was detected from the sample surface. Circularly polarized lights were discriminated by using a quarter-wave plate combined with a linear polarizer, which was placed in front of the spectrometer entrance slit. A mode-locked Ti:Sapphire pulsed laser with a repetition rate of $80 \mathrm{MHz}$ and a pulse width $<100 \mathrm{fs}$ was used as the excitation source. The excitation energy was tuned to $1.55 \mathrm{eV}$, which is slightly above the band edge of the GaAs barrier. The diameter of the excitation laser spot was approximately $0.1 \mathrm{~mm}$. Note that the PL intensity obtained by time-resolved measurements is significantly decreased at energies less than $1.40 \mathrm{eV}$ due to the detection limit of our streak camera. The calibration for the detection sensitivity has been performed in this study. Here, the characteristic of the detection sensitivity at energies less than $1.30 \mathrm{eV}$ was estimated from the extrapolation method due to no data. In terms of the time-dependent decay time and circular polarization degree (CPD) transients of QD-PL, we discuss the spin transfer within QNCs. Here, the CPD is defined as $\left(I_{\sigma+}-I_{\sigma-}\right) /\left(I_{\sigma+}+I_{\sigma-}\right)$, where $I_{\sigma \pm}$ denotes the $\sigma \pm$-polarized PL intensities. The initial CPD generated in the GaAs layers is expected to be $50 \%$ according to the optical selection rule. ${ }^{16}$ The CPD measured in QDs reflects the polarization of electron spins. ${ }^{1}$

Figure 1(a) shows a cross-sectional TEM image of a QNC consisting of 9 layers of QDs. The QD diameter monotonically increases from $25 \mathrm{~nm}$ at the first layer to $45 \mathrm{~nm}$ at the ninth layer. The
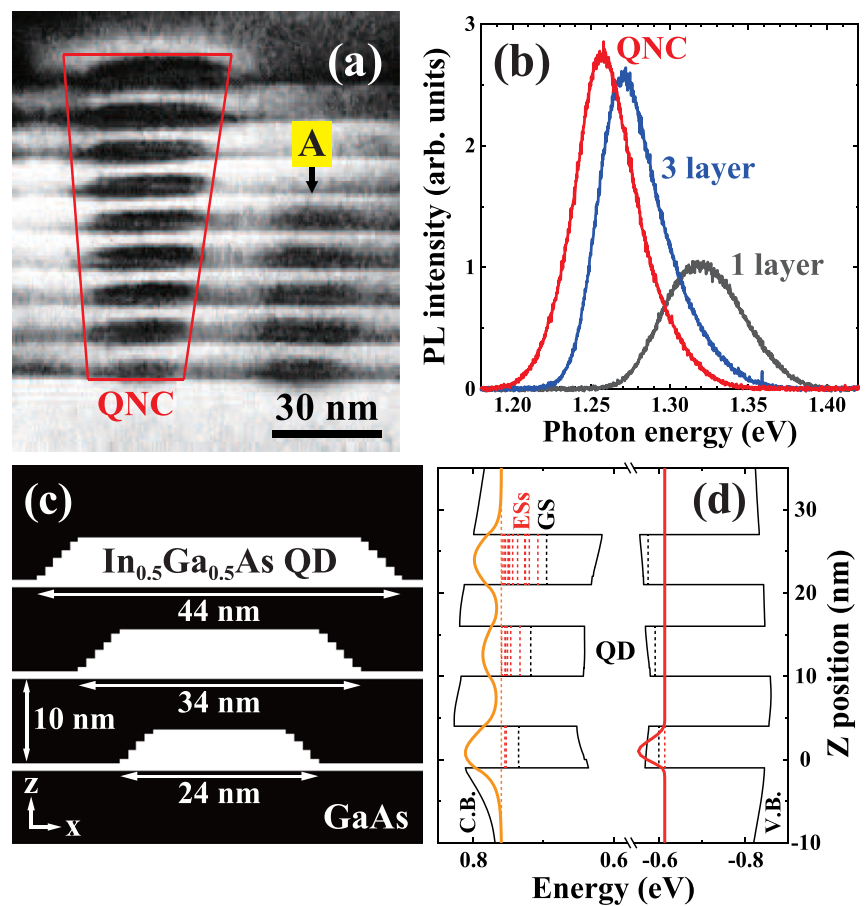

FIG. 1. (a) Cross-sectional TEM image of a QNC. The red line shows the increase in the QD size toward the top layer. Label A indicates the 5-layer stacked QDs. (b) PL spectra of the QD ground state for three samples. (c) Calculation model of sizemodulated three layers of QDs. (d) Wavefunction calculation for the simulated model (c). The black and red dashed lines show the ground state (GS) and excited states (ESs) for each QD. The orange and red solid lines show the coupled excited state of electron and the typical excited state of hole. corresponding increase was not observed for 3 layers of QDs. From the TEM image, 5-layer stacked QDs are also observed (see label A). The growing QD size reduces the areal QD density, ${ }^{13}$ leading to inhomogeneous QD stacking. From an overview TEM image, a large number of QNCs were observed, whereas the ratio of such incompletely stacked QDs was estimated to be 15\% (see Fig. S1). The detailed TEM analysis also indicates the decrease in the wetting layer thickness toward the top QD layer, which can be ascribed to the earlier formation of upper QDs due to the accumulation of the strain field induced by the underlying QDs (see Fig. S2). The AFM analysis shows the areal QD densities of 2.4-3.2 $\times 10^{10} \mathrm{~cm}^{-2}$ and the averaged center-to-center distance of approximately $60 \mathrm{~nm}$ for the three samples. This QD separation means that there is no electronic coupling at excited states among laterally aligned QDs according to the calculation result. ${ }^{9}$ When the center-to-center distance is larger than $40 \mathrm{~nm}$, electron wavefunctions are not coupled among QDs.

Figure 1(b) shows the PL spectra of the QD ground state (GS) at $6 \mathrm{~K}$ for three samples detected with a charge-coupled device sensitive to lower energies. In comparison to the single layer of QDs, centered at $1.32 \mathrm{eV}$, the PL peak shifts to lower energy by $47 \mathrm{meV}$ for 3 layers and shifts additional $14 \mathrm{meV}$ for QNC. Since the lateral QD structure is identical among the three samples, we attribute the lower energy shift of the PL to the reduction of quantum size effects due to the increase in the effective QD heights. ${ }^{18}$ The slight shift of the PL peak for QNC relative to 3 layers can be due to the fact that the PL emission mainly originates from the smaller-sized QD (lower QD) layers of QNC, as described below. The integrated PL intensity for 3 layers is 2.3 times higher than that for a single layer. The increase in the PL intensity can be attributed to the increase in the number of QDs contributing to the PL emission. However, note that there is a slight increase in the PL intensity from 3 to 9 layers (QNC). Figures 1(c) and 1(d) show the simplified model of a size-modulated QNC, where the layer number is set to three, and the calculation results of eigenstates, and the corresponding wavefunctions using the nextnano software package. ${ }^{19}$ In this simulation, the influence of strain on the band structures is taken into account based on the previous report of calculations of strained InAs/GaAs QDs. ${ }^{20}$ The presence of strain leads to an increase in the local band edge in the close vicinity of the QDs. ${ }^{21}$ Hole wavefunctions are strongly localized at the single QD layer due to the higher effective mass, whereas electron wavefunctions are delocalized in the stacking direction due to the lower effective mass. Such spatial separation of electron and hole wavefunctions can reduce the emission intensity. Here, the penetration depth of the excitation light with an excitation energy of $1.55 \mathrm{eV}$ is estimated to be $750 \mathrm{~nm}$ based on the previous report. ${ }^{22} \mathrm{~A}$ large number of carriers were generated in the $400 \mathrm{~nm}$-thick GaAs layer (depth of 120-520 nm from the sample surface) below the first QD layer. Electrons are likely to be injected into the upper QD layers with lower potentials via coupled excited states, whereas holes are likely to be localized at the lower QD layers near the dominant generation layer of carriers. Therefore, we anticipate that the dominant emission comes from the lower QD layers. This calculation also indicates the increase in the number of excited states toward the upper QD layer.

Figure 2(a) shows the circularly polarized time-integrated PL spectra and the corresponding CPD at the excitation power of $4 \mathrm{~mW}$ for 3 layers of QDs and QNC. The QNC demonstrated a much higher CPD value of $68 \%$ at the excited states, indicated by the yellow 

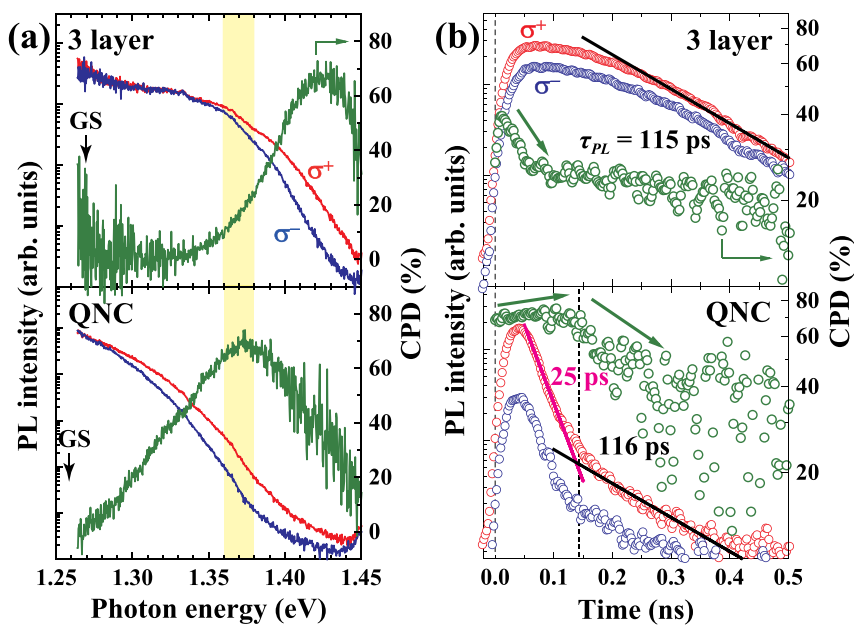

FIG. 2. (a) Circularly polarized time-integrated PL spectra; (b) PL time profiles and corresponding CPD from the excited states indicated by the rectangles in (a) for 3 layers of QDs (above) and QNC (below) at an excitation power of $4 \mathrm{~mW}$. The black arrows in (a) indicate the ground state (GS). The solid lines in (b) show the results of single exponential decay fitting for the PL decay. The black dashed line indicates the time position, where the CPD stops increasing and subsequently decays with time.

rectangle, compared to the $\mathrm{CPD}$ of $17 \%$ for 3 layers. Here, the excitation power dependence of CPD spectra showed a clear difference between two samples particularly in the energy range from 1.36 to $1.38 \mathrm{eV}$ indicated by the yellow rectangle (see Fig. S3). For the 3 layers of QDs, the CPD value averaged in the abovementioned energy range significantly decreased from $69 \%$ at $2 \mathrm{~mW}$ to $1 \%$ at $10 \mathrm{~mW}$, which is due to the spin-state filling effect in QDs. ${ }^{7,8}$ In contrast, for the QNC, the averaged CPD value was almost unchanged from $45 \%$ at $2 \mathrm{~mW}$ to $46 \%$ at $10 \mathrm{~mW}$, although the value increased from $45 \%$ at $2 \mathrm{~mW}$ to $75 \%$ at $5 \mathrm{~mW}$, as discussed in Fig. 4(a). To reveal the origin of the excitation-power-independent high spin polarization for QNC, the abovementioned energy range was focused in this study. Taking into account the width of the analyzed energy range and the diameter of the excitation laser spot described above, QD ensembles of various sizes were excited. Therefore, we expect that the analyzed energy range corresponds to the ensemble excited states of QDs, which are electronically coupled among vertically aligned QDs as shown in Fig. 1(d).

Figure 2(b) shows the circularly polarized PL time profiles and the corresponding CPDs from the excited states indicated by the yellow rectangle in Fig. 2(a). For 3 layers, a drastic decrease in CPD from $40 \%$ to $25 \%$ was observed in the initial time region. This CPD decrease is caused by the spin-state filling effect. ${ }^{7,8}$ In contrast, the CPD value for QNC increased from $70 \%$ to $75 \%$. Here, an intradot relaxation cannot contribute to this CPD increase. This relaxation process mainly contributes to the initial CPD increase from 0 to $\sim 30$ ps with a relaxation time shorter than $1 \mathrm{ps}^{23}$ In this study, the longer time scale increase in the CPD value with the time range longer than $100 \mathrm{ps}$ is mainly focused on. As indicated by the black dashed line, after the CPD stopped increasing, a conventional spin relaxation with a time constant of $0.6 \mathrm{~ns}$ was seen. ${ }^{5,7,8}$ The large CPD fluctuation seen after $0.25 \mathrm{~ns}$ is attributed to the low $\mathrm{S} / \mathrm{N}$ ratio originating from the weakness of the net PL intensity in this time region. Interestingly, we can see a biexponential PL decaying feature for the QNC, i.e., a faster decay time of 25 ps and a slower decay time of 116 ps. The increase in coupled QDs makes additional decay channels, which makes the PL decay time becomes faster. Therefore, we attribute the much faster decaying to the interdot relaxation of carriers within QNCs, which is due to the strong electronic coupling among vertically aligned QDs. In contrast, the slower decay time of $116 \mathrm{ps}$ is close to the $115 \mathrm{ps}$ for 3 layers, which means that the slower decay is derived from stacked QDs of few layers.

We next discuss the spin transfer within QNCs and its effect on the spin-polarized emission properties. Figure 3(a) shows the same result as the QNC one in Fig. 2(b). To extract the transient CPD of QNC, we performed rate-equation analysis. The red and blue solid lines in Fig. 3(a) show the best-fitted simulation results of $\sigma^{+}$- and $\sigma^{-}$ -PL transients, which are decomposed into two components with faster decay (orange dashed line) and slower decay (green dashed line), respectively. This simulation result can fit well the transient CPD (see black solid line), enabling us to decompose the overall transient CPD into that for QNC (orange solid line) and that for fewer-layer stacked QDs (green solid line). The QDs show a conventional CPD decrease from $65 \%$ to $23 \%$ for $0.5 \mathrm{~ns}$. In contrast, the QNC shows a CPD increase from $67 \%$ to $76 \%$ in the time region of $0-0.12$ ns and subsequently a constant value, although no emission occurred after 0.25 ns. Figure 3 (b) shows the circularly polarized PL time profiles and the corresponding CPD from QD ES indicated by the yellow rectangle in Fig. 2(a), measured at the higher excitation power of $12.5 \mathrm{~mW}$. In this case, biexponential PL decaying disappears. With increasing excitation power, the PL decay time for QNC becomes slower due to the suppression of the interdot relaxation via the decrease in decay



FIG. 3. Circularly polarized PL time profiles and corresponding CPD from QD ES at excitation powers of (a) 4 and (b) $12.5 \mathrm{~mW}$ for QNC. The red and blue solid lines in (a) show the best-fitted simulation results of transient PL, which are decomposed into two components for QNC (faster decay) and QDs (slower decay). The black, orange, and green solid lines in (a) show the corresponding transient CPDs. The solid lines in (b) show the best-fitted results of rate equations considering an effective spin transfer time among QDs, $\tau_{\mathrm{tr}}^{\text {eff }}$, which was defined in a previous report. ${ }^{2}$ Simulation results of transient CPD for $\tau_{\mathrm{tr}}^{\text {eff }}=50,100$, and 300 ps are also shown. 
channels. As a result, the emission from QNC becomes dominant in the time region of $0-0.5 \mathrm{~ns}$, although in the case of $4 \mathrm{~mW}$, that in the time region of $0-0.15 \mathrm{~ns}$ is dominant. The transient CPD shows a clear increase from $45 \%$ to $55 \%$ for $0.2 \mathrm{~ns}$. Such spin amplification is expected for strongly coupled QDs. ${ }^{23}$ Rate equation simulations, which take into account an effective spin transfer time among QDs, $\tau_{\text {tr }}^{\text {eff }}$, indicate that a CPD increase occurs when $\tau_{\text {tr }}^{\text {eff }}<50 \mathrm{ps}^{24}$ The solid lines in Fig. 3(b) show the best-fitted results of rate equations considering the parameter $\tau_{\mathrm{tr}}^{\text {eff }}$. The obtained $\tau_{\mathrm{tr}}^{\text {eff }}=36$ ps can explain the CPD amplification well, as well as the PL decaying features with both polarizations. When $\tau_{\text {tr }}^{\text {eff }}$ becomes slower, corresponding to a weaker coupling among QDs, CPD amplification disappears, and thus, CPD largely decreases, as shown in Fig. $3(\mathrm{~b})$. Here, $\tau_{\text {tr }}^{\text {eff }}=19$ ps was obtained for the QNC component in Fig. 3(a) using the abovementioned fitting analysis. The slower spin transfer with increasing excitation power comes from the suppression of the interdot relaxation described above. Comparing these transient CPD properties at the different excitation powers suggests that the spin transfer rate depends on the degree of state filling of lower-energy states of neighboring QDs, ${ }^{9}$ and a moderate condition of excited spin density is needed to realize a higher spinpolarized emission from QDs.

Figure 4(a) shows the excitation power dependence of maximum values of time-dependent CPD (CPD max) of QD ES PL for three samples. Here, the energy range from 1.36 to $1.38 \mathrm{eV}$ indicated by the yellow rectangle in Fig. 2(a) was analyzed as the QD ES for all three samples. For a single layer of QDs, the CPD max monotonically decreased from $62 \%$ at $1 \mathrm{~mW}$ to $8 \%$ at $10 \mathrm{~mW}$. This CPD decrease can be explained by considering the spin-state filling effect in QDs. For 3 layers, the CPD max increased from $59 \%$ at $1 \mathrm{~mW}$ to $81 \%$ at 2 $\mathrm{mW}$. This CPD increase is attributed to the interdot relaxation of spins in coupled QDs. ${ }^{23}$ Minority spins are selectively transferred from the emissive state to lower-energy states of neighboring QD layers. With excitation power beyond $2 \mathrm{~mW}$, CPD max largely decreases to $11 \%$ at $10 \mathrm{~mW}$. The lowest CPD max of $56 \%$ can be seen at $2 \mathrm{~mW}$ for QNC. The lower CPD at lower excitation power is caused by the interdot relaxation of both spins from the emissive state. CPD max gradually increased to $80 \%$ at $5 \mathrm{~mW}$. The mechanism of the CPD increase is the same as that for 3 layers described above. The important finding is the
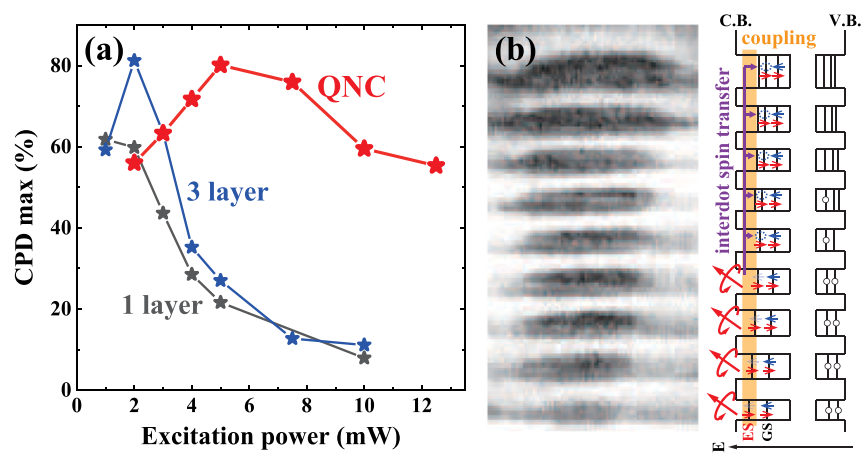

FIG. 4. (a) Excitation power dependence of maximum values of time-dependent CPD (CPD max) of QD ES PL for three samples. (b) TEM image of a sizemodulated QNC. Schematic model of spin-polarized emission from lower-lying QD layers induced by selective transfer of minority spins to upper-lying nonemissive layers via interdot coupling of electron wavefunctions. suppression of the CPD decrease with increasing excitation power. The CPD max of $60 \%$ is obtained even at $10 \mathrm{~mW}$. Spin transfer within QNCs can effectively suppress the spin-state filling effect at the QD emissive state. The significant spin transfer is evidenced by $\tau_{\text {tr }}^{\text {eff }}$ $=36$ ps, as shown in Fig. 3(b). As discussed above, since the emission predominantly comes from the lower QD layers of QNC, the upper QD layers with lower potentials behave as the nonemissive receiver of minority spins, as shown in Fig. 4(b). Furthermore, the upper layers have abundant excited states, where electron spins are transferred, as shown in Fig. 1(d). The large number of relaxation channels for minority spins leads to the high spin polarization at coupled excited states independent of excitation-spin density. Therefore, the combination of QD size modulation and electronic coupling in the stacking direction can significantly enhance the spin polarization at lower QD layers due to the selective transfer of minority spins to the upper layers. In comparison to laterally coupled QDs, ${ }^{9}$ which show a CPD max of $70 \%$, the QNC is more attractive for higher spin-polarized emission from QDs. This prominent optical-spin property is attributed to the significant spin transfer within QNCs originating from the smaller separation between QDs. These findings imply a need to utilize a spin amplification at QD excited states for the application of QDs to optospintronic devices. For the application to spin-polarized laser diodes, it is needed that a high spin polarization at emissive states can be kept even at high excited spin density. The QNC is advantageous as an active layer of optospintronic devices because of the maintained high spin polarization in a wide range of excited spin densities. In addition, the QNC has an implication in excited spin engineering because the spin transfer rate can be controlled by changing the spacer thickness.

In this study, spin transfer within a size-modulated QNC composed of 9 layers of QDs was investigated. The QNC shows a temporal spin amplification higher than $80 \%$ at coupled excited states. This high spin polarization is attributed to the selective transfer of minority spins from the lower-lying emissive layers to the upper-lying nonemissive layers, where holes cannot be transferred due to the higher effective mass. The QNC can also retain high spin polarization even at high excitation spin density. The findings of this study offer significant insights into the future development of spin-functional optical devices that use QNCs as optically active layers.

See the supplementary material for the detailed TEM analysis of QNCs and the excitation power dependence of CPD spectra for 3 layers of QDs and QNC.

This study was supported by the Japan Society for the Promotion of Science, Grant-in-Aid for Scientific Research (S) (KAKENHI No. 16H06359) and Grant-in-Aid for Young Scientists (KAKENHI No. 19K15380), and Hattori Hokokai Foundation.

\section{REFERENCES}

${ }^{1}$ C. H. Li, G. Kioseoglou, O. M. J. van't Erve, M. E. Ware, D. Gammon, R. M. Stroud, B. T. Jonker, R. Mallory, M. Yasar, and A. Petrou, Appl. Phys. Lett. 86, 132503 (2005).

${ }^{2}$ S. Hövel, N. C. Gerhardt, M. R. Hofmann, F.-Y. Lo, D. Reuter, A. D. Wieck, E. Schuster, W. Keune, H. Wende, O. Petracic, and K. Westerholt, Appl. Phys. Lett. 92, 242102 (2008).

${ }^{3}$ L. Zhu, W. Huang, P. Renucci, X. Marie, Y. Liu, Y. Li, Q. Wu, Y. Zhang, B. Xu, Y. Lu, and Y. Chen, Phys. Rev. Appl. 8, 064022 (2017). 
${ }^{4}$ M. Paillard, X. Marie, P. Renucci, T. Amand, A. Jbeli, and J. M. Gérard, Phys. Rev. Lett. 86, 1634 (2001).

${ }^{5}$ A. Tackeuchi, R. Ohtsubo, K. Yamaguchi, M. Murayama, T. Kitamura, T. Kuroda, and T. Takagahara, Appl. Phys. Lett. 84, 3576 (2004).

${ }^{6}$ T. Amano, T. Sugaya, and K. Komori, Appl. Phys. Lett. 89, 171122 (2006).

${ }^{7}$ T. Kiba, X. J. Yang, T. Yamamura, Y. Kuno, A. Subagyo, K. Sueoka, and A. Murayama, Appl. Phys. Lett. 103, 082405 (2013).

${ }^{8}$ T. Yamamura, T. Kiba, X. J. Yang, J. Takayama, A. Subagyo, K. Sueoka, and A. Murayama, J. Appl. Phys. 116, 094309 (2014).

${ }^{9}$ S. Hiura, K. Takeishi, M. Urabe, K. Itabashi, J. Takayama, T. Kiba, K. Sueoka, and A. Murayama, Appl. Phys. Lett. 113, 023104 (2018).

${ }^{10}$ S. Hiura, M. Urabe, K. Takeishi, K. Itabashi, J. Takayama, T. Kiba, K. Sueoka, and A. Murayama, Semicond. Sci. Technol. 34, 025001 (2019).

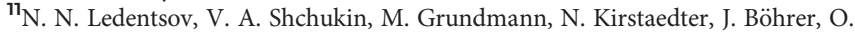
Schmidt, D. Bimberg, V. M. Ustinov, A. Yu. Egorov, A. E. Zhukov, P. S. Kop'ev, S. V. Zaitsev, N. Yu. Gordeev, Zh. I. Alferov, A. I. Borovkov, A. O. Kosogov, S. S. Ruvimov, P. Werner, U. Gösele, and J. Heydenreich, Phys. Rev. B 54, 8743 (1996).

${ }^{12}$ O. G. Schmidt, O. Kienzle, Y. Hao, K. Eberl, and F. Ernst, Appl. Phys. Lett. 74, 1272 (1999).

${ }^{13}$ Zh. M. Wang, H. Churchill, C. E. George, and G. J. Salamo, J. Appl. Phys. 96, 6908 (2004).
${ }^{14}$ B. Lita, R. S. Goldman, J. D. Phillips, and P. K. Bhattacharya, Appl. Phys. Lett. 74, 2824 (1999).

${ }^{15}$ D. M. Bruls, P. M. Koenraad, H. W. M. Salemink, J. H. Wolter, M. Hopkinson, and M. S. Skolnick, Appl. Phys. Lett. 82, 3758 (2003).

${ }^{16}$ F. Meier and B. P. Zakharchenya, Optical Orientation (North-Holland, Amsterdam, 1984).

${ }^{17}$ S. Hiura, K. Itabashi, K. Takeishi, J. Takayama, T. Kiba, and A. Murayama, Appl. Phys. Lett. 114, 072406 (2019).

${ }^{18}$ Y. Nakata, Y. Sugiyama, T. Futatsugi, and N. Yokoyama, J. Cryst. Growth 175-176, 713 (1997).

${ }^{19}$ S. Birner, T. Zibold, T. Andlauer, T. Kubis, M. Sabathil, A. Trellakis, and P. Vogl, IEEE Trans. Electron Devices 54, 2137 (2007).

${ }^{20}$ C. Pryor, Phys. Rev. B 57, 7190 (1998).

${ }^{21}$ A. Schliwa, G. Hönig, and D. Bimberg, Electronic Properties of III-V Quantum Dots (Springer International Publishing, Cham, 2014), pp. 57-85.

${ }^{22}$ D. E. Aspnes, S. M. Kelso, R. A. Logan, and R. Bhat, J. Appl. Phys. 60, 754 (1986).

${ }^{23}$ V. K. Kalevich, M. Paillard, K. V. Kavokin, X. Marie, A. R. Kovsh, T. Amand, A. E. Zhukov, Yu. G. Musikhin, V. M. Ustinov, E. Vanelle, and B. P. Zakharchenya, Phys. Rev. B 64, 045309 (2001).

${ }^{24}$ K. Takeishi, S. Hiura, J. Takayama, K. Itabashi, M. Urabe, A. Washida, T. Kiba, and A. Murayama, Phys. Rev. Appl. 10, 034015 (2018). 